\title{
A Material-Based Platform to Modulate Fibronectin Activity and Focal Adhesion Assembly
}

\author{
Frankie A. Vanterpool, ${ }^{1,2, *}$ Marco Cantini,, ${ }^{1, *}$ F. Philipp Seib, ${ }^{2}$ and Manuel Salmerón-Sánchez ${ }^{1}$
}

\begin{abstract}
We present a detailed characterization of fibronectin (FN) adsorption and cell adhesion on poly(ethyl acrylate) (PEA) and poly(methyl acrylate) (PMA), two polymers with very similar physicochemical properties and chemical structure, which differ in one single methyl group in the lateral chain of the polymer. The globular solution conformation of FN was retained following adsorption onto PMA, whereas spontaneous organization of FN into protein (nano) networks occurred on PEA. This distinct distribution of FN at the material interface promoted a different availability, measured via monoclonal antibody binding, of two domains that facilitated integrin binding to FN: $\mathrm{FNIII}_{10}$ (RGD sequence) and $\mathrm{FNIII}_{9}$ (PHSRN synergy sequence). The enhanced exposure of the synergy domain on PEA compared to PMA triggered different focal adhesion assemblies: L929 fibroblasts showed a higher fraction of smaller focal plaques on PMA (40\%) than on PEA (20\%). Blocking experiments with monoclonal antibodies against FNIII $_{10}$ (HFN7.1) and FNIII $_{9}$ (mAb1937) confirmed the ability of these polymeric substrates to modulate FN conformation. Overall, we propose a simple and versatile material platform that can be used to tune the presentation of a main extracellular matrix protein (FN) to cells, for applications than span from tissue engineering to disease biology.
\end{abstract}

Key words: biomaterials; cell culture; cellular biology; extracellular matrix

\section{Introduction}

$\mathbf{P}$ ROVIDING CELLS WITH A RELIABLE and robust microenvironment, able to control and understand their response, is a crucial issue for regenerative medicine and biological research in general. ${ }^{1}$ Traditional systems lack control of the intermediate layer of absorbed proteins that govern cell response and eventually direct cell fate. ${ }^{1,2}$ It is widely accepted that cell adhesion onto a synthetic surface is mediated by a layer of proteins previously adsorbed onto the material, either from the physiological fluids in vivo or from the culture medium in vitro. This layer may also be intentionally deposited, for example, through adsorption of extracellular matrix (ECM) proteins, or via chemical attachment to the substrate. ${ }^{1-5}$ These proteins include fibronectin (FN), vitronectin, and fibrinogen, known as the soluble matrix proteins of biological fluids. ${ }^{4}$ Cell adhesion is the first step in their response to a microenvironment, and plays a fundamental role in subsequent cell differentiation, growth, viability, and phenotypic response. ${ }^{6}$ Therefore, controlling the cellular response through a robust material-based strategy is applicable for a myriad of applications in biomedical research, spanning from regenerative medicine to disease biology.

FN is a high-molecular-weight glycoprotein that is found in both soluble and insoluble forms in extracellular fluids and connective tissues, respectively. ${ }^{7-9}$ It is a main component of the ECM and therefore a likely protein candidate whose state can be tailored to target cell response. The FN molecule is a dimer of $\sim 440 \mathrm{kDa}$, whose subunits contain three types of repeating units (FN repeats I, II, and III) that mediate interactions with other ECM proteins and cell surface receptors, and regulate FN-FN interactions (Fig. 1A). The RGD sequence, located in the FN repeat $\mathrm{III}_{10}$, is of key importance in controlling cell adhesion, with further regions, such as the PHSRN sequence (synergy site), in the FN repeat $\mathrm{III}_{9}$, promoting $\alpha_{5} \beta_{1}$ integrin binding to FN (Fig. 1B). ${ }^{9}$ Cells have evolved to recognize adhesive proteins through integrins, a family of trans-membrane heterodimers that serve as a link between the ECM and the cell actin cytoskeleton. ${ }^{11}$ Subsequent to binding, integrins cluster and develop into complexes, called focal adhesions, composed of structural (e.g., vinculin) and signaling molecules (e.g., focal

\footnotetext{
${ }^{1}$ Division of Biomedical Engineering, School of Engineering, University of Glasgow, Glasgow, United Kingdom.

${ }^{2}$ Strathclyde Institute of Pharmacy and Biomedical Sciences, University of Strathclyde, Glasgow, United Kingdom.

*These two authors contributed equally to this work.
} 
A

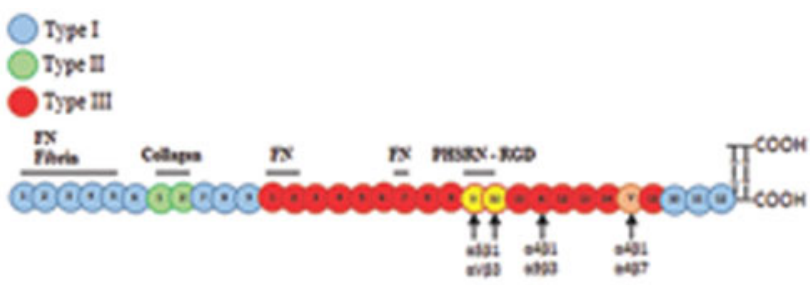

C
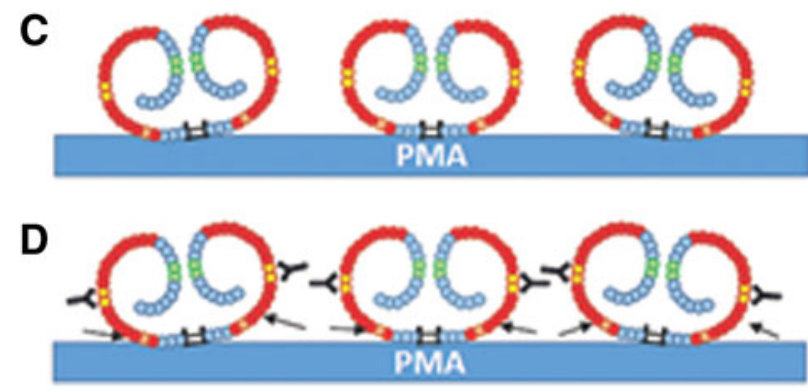

B

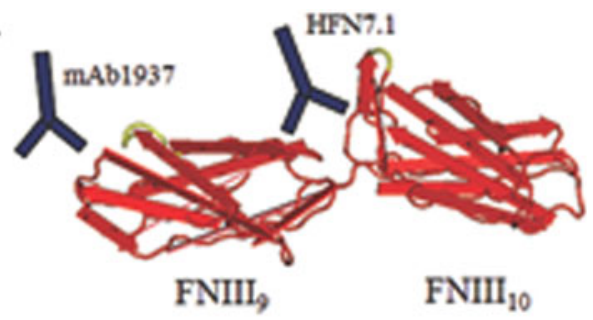

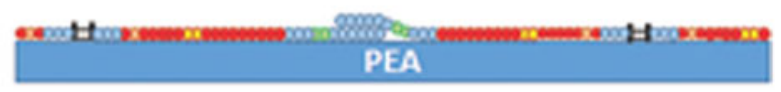

FIG. 1. Mechanistic model for the material-based modulation of FN conformation. (A) Structure of FN showing three different types of domains (I, II, and III) and binding regions for integrins. (B) Secondary structure for the main integrin binding domains of FN ( FNIII $_{9-10}$ ) highlighting the RGD and PHSRN sequences in yellow along the specific binding sites for HFN7.1 and mAb1937 antibodies, adapted from PBD ID: 1FNF. ${ }^{10}$ (C) FN takes on a globular conformation on PMA (left), whereas a (nano)network is formed on PEA (right) via the $\mathrm{FNI}_{1-5}$ domains. (D) After blocking the cell binding and/or synergy domains $\left(\right.$ FNIII $\left._{9-10}\right)$, no other integrin binding domains are accessible by cells on PMA (left), whereas they are still accessible on PEA (right). FN, fibronectin; PEA, poly(ethyl acrylate); PMA, poly(methyl acrylate).

adhesion kinase). These plaques anchor cells to the surface and their formation triggers cell response. ${ }^{12}$ Overall, FN adopts a fundamental role in mediating and promoting cell adhesion, and in regulating cell survival and phenotypic response.

Several studies have demonstrated that surface chemistry is a key parameter that can alter the amount and conformation of adsorbed FN. ${ }^{13-20}$ For example, using differently terminated self-assembled monolayers (SAMs), García and coworkers demonstrated that hydrophilic surfaces $(\mathrm{OH}-$ SAM) induced the least amount of structural change in FN or in the recombinant fragment $\mathrm{FNIII}_{7-10}$, while hydrophobic $\mathrm{CH}_{3}$-SAM provoked the largest structural changes. ${ }^{13,14}$ Surface chemistry can therefore be used to manipulate the state and activity of the protein through simple physical adsorption. We have previously demonstrated that poly(ethyl acrylate) (PEA) surfaces induce the organization of FN into physiological-like fibrillar (nano) networks upon adsorption onto the polymeric substrate. ${ }^{20,21}$ However, the removal of a methyl group from the monomer [i.e., using poly(methyl acrylate) (PMA) instead] prevented this phenomenon, leading to $\mathrm{FN}$ adsorption in a globular conformation. In this work, we characterized the state of the protein on these surfaces in relation to protein concentration and surface chemistry. We used L929 fibroblast, plated on engineered FN substrates, and determined the cellular response in relation to the presentation of FN at the material interface. Overall, we intended to establish a simple and robust tissue culture platform that enables us to control the state of the intermediate adsorbed protein layer, thereby tailoring the microenvironment presented to cells (Fig. 1). We propose that this material-based platform is a versatile tool to both direct and elucidate mechanisms underlying cell response for applications spanning from tissue engineering to disease biology.

\section{Materials and Methods}

\section{Samples preparation}

PEA and PMA polymers were synthetized by radical polymerization of ethyl acrylate and methyl acrylate (Sigma, St. Louis, MO), initiated by benzoin at $1 \mathrm{wt} \%$ and $0.35 \mathrm{wt} \%$, respectively. Samples were then dried by vacuum extraction to constant weight and solubilized in toluene $2.5 \% \mathrm{w} / \mathrm{v}$ for PEA and 6\% w/v for PMA. PEA and PMA solutions were then spin coated onto $12 \mathrm{~mm}$ round glass coverslips for $30 \mathrm{sec}$ at a velocity of 2000 and $3000 \mathrm{rpm}$, respectively, with an acceleration of $3000 \mathrm{rpm} / \mathrm{sec}$. Excess toluene was removed by placing coverslips under the vacuum for $2 \mathrm{~h}$ at $60^{\circ} \mathrm{C}$.

\section{Protein adsorption}

Human plasma FN (Sigma) solutions, in Dulbecco's phosphate buffered saline (DPBS) at 2, 5, 10, and $20 \mu \mathrm{g} / \mathrm{mL}$, were coated onto PEA and PMA coverslips for $1 \mathrm{~h}$.

\section{Water contact angle}

Water contact angle analysis was carried out on PEA or PMA surfaces alone and on FN-coated surfaces. For each condition, the static contact angle (SCA), advancing contact angle (ACA), and receding contact angle (RCA) were determined $(n=9)$. SCA was determined by placing a drop of $3 \mu \mathrm{L}$ of water on the surface using a needle and recording the images at 12 frames/sec for $30 \mathrm{sec}$ while measuring the angle of the drop with the polymer surface. ACA was determined by placing the needle in the previously deposited drop and progressively adding water in order to observe an increase in the length of the baseline. RCA was determined by progressively removing the water with the needle until the drop was removed. RCA is the angle at which the baseline starts 
decreasing. Measurements were carried out using a Theta optical tensiometer (Biolin Scientific, Stockholm, Sweden).

\section{Immunostaining of FN}

Immunostaining was done with polyclonal anti-FN (Millipore, Darmstadt, Germany) primary antibody directed against FN. PEA and PMA samples were coated with FN at different concentrations for $1 \mathrm{~h}$. Freshly prepared samples were fixed with formaldehyde $3.7 \% \mathrm{v} / \mathrm{v}$ for $30 \mathrm{~min}$ at $4^{\circ} \mathrm{C}$ and then washed with PBS. Samples were incubated with anti-FN (1:400 dilution in PBS/BSA $1 \% \mathrm{w} / \mathrm{v})$ for $1 \mathrm{~h}$ at room temperature and then washed twice with DPBS/ Tween-20 $0.5 \% \mathrm{v} / \mathrm{v}$. Next, samples were incubated with Cy3 anti-mouse secondary antibody (1:200 in PBS/BSA 1\% $\mathrm{w} / \mathrm{v})$ for $1 \mathrm{~h}$ at room temperature in the dark and then washed twice and mounted with vectashield (Vector Laboratories, Inc., Burlingame, CA) and imaged using an epifluorescence microscope (Zeiss AXIO Observer Z1, Jena, Germany).

\section{Atomic force microscopy}

Several areas of the surface were scanned at different area sizes and line rates: $(5 \times 5) \mu \mathrm{m}^{2}(0.5 \mathrm{~Hz}) ;(2 \times 2) \mu \mathrm{m}^{2}$ $(0.7 \mathrm{~Hz}) ;(1 \times 1) \mu \mathrm{m}^{2}(1 \mathrm{~Hz}) ;(0.5 \times 0.5) \mu \mathrm{m}^{2}(1 \mathrm{~Hz})$. The surface height, lock-in phase, and lock-in amplitude were observed in tapping-mode atomic force microscopy (AFM; Nanowizard 3 from JPK, Berlin, Germany) using cantilevers with a force constant of $3 \mathrm{~N} / \mathrm{m}$, a resonance frequency of $75 \mathrm{kHz}$, and a pyramidal tip, with radius of curvature less than $8 \mathrm{~nm}$ (MPP-21120 from Bruker, Billerica, MA).

\section{Micro-bicinchoninic acid protein quantification}

PEA, PMA, and glass coverslips were coated with FN for $1 \mathrm{~h}$ at different solution concentrations and the density of adsorbed protein was determined by measuring the amount of nonadsorbed FN. A stock solution of FN was diluted at $20,10,5$, and $2 \mu \mathrm{g} / \mathrm{mL}$ to determine the exact concentration of the stock FN solution and the amount of FN deposited onto the coverslips. After coating for $1 \mathrm{~h}$ the $\mathrm{FN}$ solution was collected and transferred to a 96-well plate followed by the addition of the bicinchoninic acid working reagent (Thermo Fisher Scientific, Waltham, MA). The plate was then shaken for $30 \mathrm{sec}$, covered, and placed in an incubator for $2 \mathrm{~h}$ at $37^{\circ} \mathrm{C}$ and then absorbance was read at $562 \mathrm{~nm}$ with a Tecan NanoQuant Infinite M200 Pro plate reader (Männedorf, Switzerland).

\section{Enzyme-linked immunosorbent assay}

Enzyme-linked immunosorbent assay (ELISA) was performed on PEA and PMA coated with different concentrations of FN using primary monoclonal antibodies HFN7.1 (DSHB, Iowa City, IA) and mAb1937 (Millipore) combined with HRP-Goat Anti-Mouse (Invitrogen, Carlsbad, CA) secondary antibody, which is coupled with a peroxidase for absorbance reading. FN was adsorbed at 20 and $1 \mu \mathrm{g} / \mathrm{mL}$ as well as a control without protein on PEA and PMA for $1 \mathrm{~h}$. Samples were washed with PBS, transferred to new 24well plates, and then blocked with PBS/BSA $1 \%$ w/v for $30 \mathrm{~min}$ at room temperature. Subsequently, samples were in- cubated with the primary antibodies for $1 \mathrm{~h}$ at room temperature and washed with PBS/Tween-20 0.5\% v/v. The secondary antibody (HRP-Goat Anti-Mouse) was then added on all samples for $1 \mathrm{~h}$ at room temperature in the dark and washed with PBS/Tween-20 0.5\% v/v. The substrate (color reagents A and B from R\&D Systems, Minneapolis, MN) was then added for $20 \mathrm{~min}$ at room temperature, in the dark, stopped with a stop solution (R\&D Systems), and transferred to a 96-well plate and absorbance was read at 450 and $550 \mathrm{~nm}$.

\section{Cell culture}

Mouse L929 fibroblasts (ECACC-85011425, London, United Kingdom) were thawed and re-suspended in Dulbecco's modified Eagle's medium (with $4.5 \mathrm{~g} / \mathrm{L}$ glucose and Lglutamine) with $1 \% \mathrm{v} / \mathrm{v}$ penicillin/streptomycin and $10 \% \mathrm{v} / \mathrm{v}$ fetal bovine serum (FBS; Life Technologies, Carlsbad, CA). Cells were grown at $37^{\circ} \mathrm{C}, 5 \% \mathrm{CO}_{2}$, and harvested by trypsinization at $90 \%$ confluency.

\section{Attachment assay}

This was done according to the protocol by Humphries. ${ }^{22}$ UV-sterilized PEA and PMA samples were coated with FN $(20 \mu \mathrm{g} / \mathrm{mL})$ for $1 \mathrm{~h}$ and washed twice with PBS. Samples were then blocked with heat-denatured BSA $1 \%$ w/v (Roche, Basel, Switzerland) for $30 \mathrm{~min}$ at room temperature. During this period, cells were harvested, trypsinized, and resuspended in complete medium (containing 10\% FBS). The cell suspension was then incubated for $10 \mathrm{~min}$ at $37^{\circ} \mathrm{C}, 5 \%$ $\mathrm{CO}_{2}$.

Cells were seeded onto the surfaces at a seeding density of $8.5 \times 10^{4}$ cells $/ \mathrm{cm}^{2}$ for $20 \mathrm{~min}$ at $37^{\circ} \mathrm{C}, 5 \% \mathrm{CO}_{2}$. Surfaces were washed twice with PBS to remove cells that were not firmly attached, fixed with formaldehyde $3.7 \%$ for $20 \mathrm{~min}$ at $4^{\circ} \mathrm{C}$, and washed again. Samples were permeabilized for 5 min using a Triton X-100 based permeabilization buffer $(0.5 \% \mathrm{v} / \mathrm{v}$ Triton X-100, $10.3 \mathrm{w} / \mathrm{v} \%$ saccharose, $0.292 \% \mathrm{w} /$ $\mathrm{v} \mathrm{NaCl}, 0.06 \%$ w/v $\mathrm{MgCl}_{2}$, and $0.476 \%$ w/v HEPES adjusted to $\mathrm{pH}$ 7.2) and mounted with vectashield with DAPI to stain the nuclei. Images were taken using an inverted epifluorescence microscope and the number of cells attached was determined using CellC total cell count analysis (Zeiss AXIO Observer Z1).

In the case of blocking cell binding domains on FN, the same procedures were carried out using only samples coated with $20 \mu \mathrm{g} / \mathrm{mL}$ of FN and blocking the RGD and PHSRN sequences with HFN7.1 and mAb1937 at a 1:1 molar ratio $(14.6 \mu \mathrm{g} / \mathrm{mL})$ for $1 \mathrm{~h}$ at room temperature.

\section{Early adhesion assay}

PEA- and PMA-coated coverslips were sterilized for 20 min using a UV lamp and coated with FN at different concentrations $(2,5,10$, and $20 \mu \mathrm{g} / \mathrm{mL})$ for $1 \mathrm{~h}$. Samples were then washed twice with PBS and seeded with cells at $1 \times 10^{4}$ cells per well for $3 \mathrm{~h}$. After washing with PBS, samples were fixed with formaldehyde $3.7 \%$ for $20 \mathrm{~min}$ at $4^{\circ} \mathrm{C}$ and washed again before being stored in DPBS at $4^{\circ} \mathrm{C}$ until ready to use. In the case of cell adhesion after blocking specific FN domains, PEA and PMA were incubated for $1 \mathrm{~h}$ with $150 \mu \mathrm{L}$ of HFN7.1 and/or mAb1937 at a 1:1 molar ratio. 
Antibodies were diluted in $1 \%$ BSA w/v. Samples coated with $20 \mu \mathrm{g} / \mathrm{mL}$ of FN only were then permeabilized (as described above) and stained for $1 \mathrm{~h}$ using a primary antibody against mouse vinculin hVIN-1 (Sigma-Aldrich, St. Louis, MO), which stains the focal adhesions. The secondary antibody (Cy3 anti-mouse), coupled with actin staining Phallacidin, was then added for $1 \mathrm{~h}$ in the dark.

Samples were then mounted with vectashield with DAPI to stain the nuclei and visualized using an epifluorescence microscope. Images were taken and channels merged using ImageJ (1.47v) to localize nuclei, actin, and focal adhesions. The latter was quantified using the focal adhesion analysis server. ${ }^{23}$ Data such as focal adhesion size, area, and distance from the center of the cell were obtained from the server and used to calculate the geometric moment of inertia $(I)$ of cells

$$
I=\sum_{i} a_{i} r_{i}^{2}
$$

where $a_{i}$ is the area of the $i$ th focal adhesion and $r_{i}$ is the distance between the $i$ th focal adhesion and the center of the distribution of focal adhesions. Cell morphology, describing shape, was analyzed using ImageJ $(1.47 \mathrm{v})$.

\section{Statistical analysis}

Two-way ANOVA was used for the comparison of the different groups using a Bonferroni post hoc test to compare all columns (GraphPad Prism 5.03) and the differences between groups were considered significant for $p<0.05$. All error bars were standard deviation.

\section{Results}

\section{FN adsorption}

Material substrates used in this study were chemically similar, consisting of a vinyl backbone chain with side group $-\mathrm{COO}\left(\mathrm{CH}_{2}\right)_{x} \mathrm{H}$, with $x=1$ for PMA and $x=2$ for PEA. The difference of only one methyl group in the side group yielded substrates with similar physicochemical properties $^{24}$ (Fig. 2A). Both surfaces are classified as hydrophobic, with a static contact angle of $75^{\circ}$ (Fig. 2A,D), and similar behavior in dynamic contact angles, although PEA showed more hysteresis as defined by the difference between advancing and receding angles (Fig. 2D). Contact angle hysteresis has been interpreted as a measure of molecular mobility for surfaces that are chemically homogeneous and flat. ${ }^{25,26}$

Spin-coated samples were smooth $\left(R_{\mathrm{rms}}<1 \mathrm{~nm}\right)$ and homogeneous, with a thickness of $\sim 1 \mu \mathrm{m}$. When the surfaces were coated with FN from solution concentrations of 2, 5, 10 , and $20 \mu \mathrm{g} / \mathrm{mL}$, the static and advancing contact angles were maintained, while the hysteresis increased for both polymers due to a decrease of receding angles, reaching a plateau for concentrations higher than 2 and $5 \mu \mathrm{g} / \mathrm{mL}$ for PMA and PEA, respectively (Fig. 2D). Furthermore, contact angle hysteresis of FN-coated samples was higher on PEA (e.g., $\sim 90^{\circ}$ compared to $\sim 65^{\circ}$ on PMA, for concentrations greater than $5 \mu \mathrm{g} / \mathrm{mL}$ ), suggesting a different state of the adsorbed protein layer. Immunostaining of FN adsorbed onto the polymeric substrates resulted in a different distribution of the protein at the microscale; FN forms densely packed networks on PEA, while globular aggregates appear
A
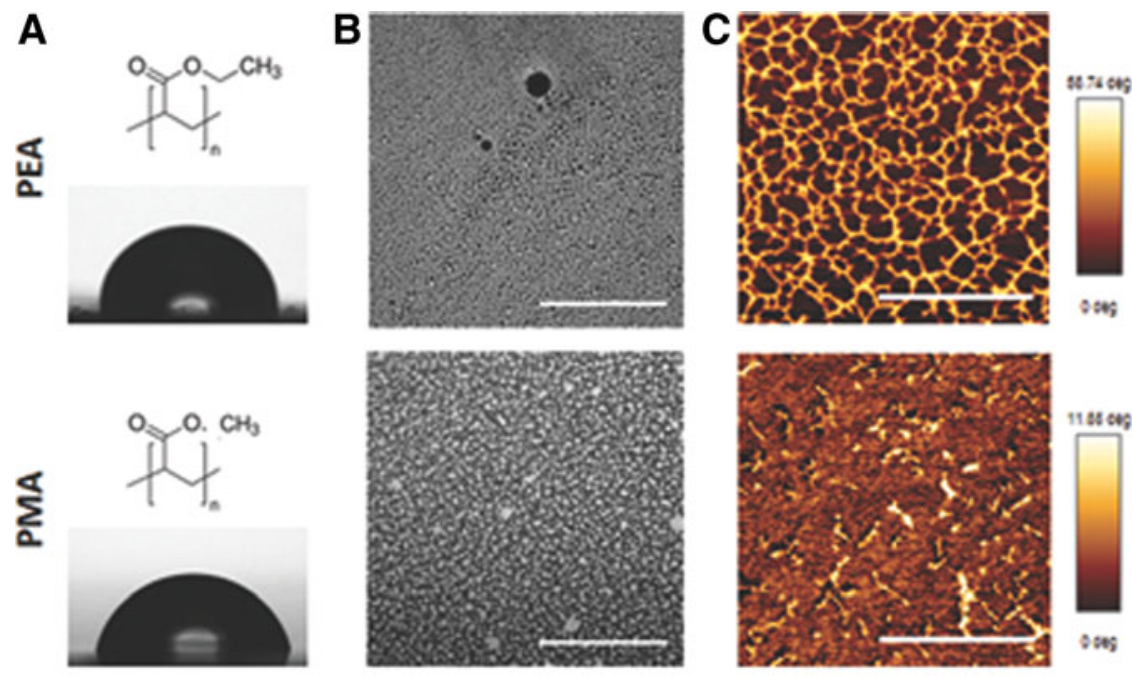

D

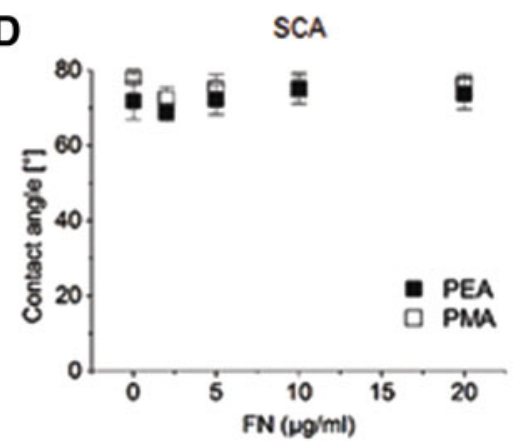

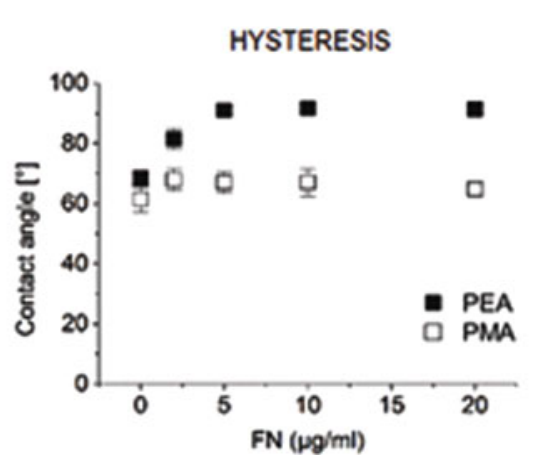

FIG. 2. Characterization of FNcoated PEA and PMA surfaces. (A) Chemical structure of polymers and water drop ( $3 \mu \mathrm{L})$ on PEA and PMA. (B) Immunostaining of FN after adsorption from a solution concentration of $20 \mu \mathrm{g} / \mathrm{mL}$ (scale bar: $50 \mu \mathrm{m}$ ). (C) Typical AFM images of FN on PEA and PMA after adsorption from a solution of concentration $20 \mu \mathrm{g} / \mathrm{mL}$ (scale bar: $0.5 \mu \mathrm{m}$ ). (D) SCA and hysteresis (difference between advancing and receding contact angles) on PEA and PMA coated from solutions with concentrations of $2,5,10$, and $20 \mu \mathrm{g} / \mathrm{mL}$ FN. AFM, atomic force microscopy; SCA, static contact angle. 

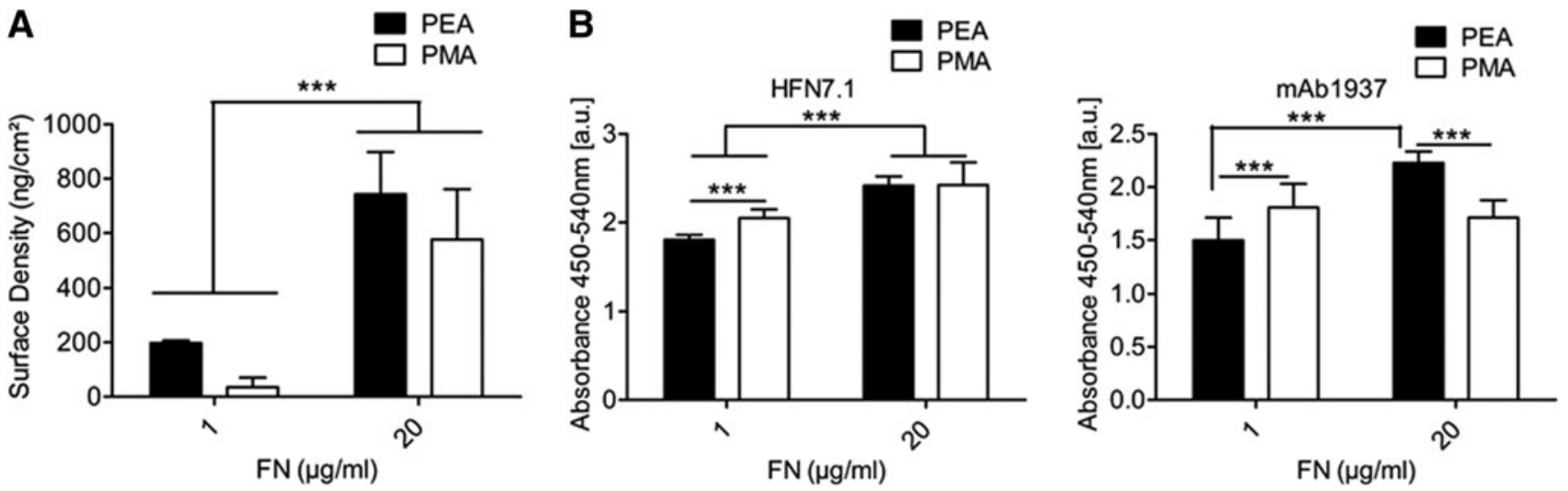

FIG. 3. FN adsorption and domain availability on PEA and PMA. (A) Surface density of FN on PEA and PMA coated from solutions of 1 and $20 \mu \mathrm{g} / \mathrm{mL}$ of FN. (B) Availability of the RGD (located within FNIII 9 ) cell binding domain (left) and the PHSRN (located within FNIII ${ }_{10}$ ) synergy domain (right) on PEA and PMA coated from solutions of concentration 1 and $20 \mu \mathrm{g} / \mathrm{mL}$ of FN. Monoclonal antibodies against these two domains of FN HFN7.1 (FNIII 10 ) and mAb1937 (FNIII $_{9}$ ) were used. $* * p<0.01, * * * p<0.001$.

on PMA (Fig. 2B). The different FN distributions were maintained regardless the concentration of the protein solution (Supplementary Fig. S1). AFM images confirmed structural differences of $\mathrm{FN}$ at the nanoscale (Fig. 2C). PEA was seen to unfold the FN arms that led to interconnected nanofibrils; in contrast, PMA surfaces promoted FN adsorbed in its globular conformation, confirming our previous results. ${ }^{21,27}$

The surface density of adsorbed protein was indirectly quantified by measuring the depletion of FN from solution. The surface density of adsorbed FN increased with solution concentration (Fig. 3A), though it remained constant between surfaces, with no statistically significant differences (e.g., $\sim 750 \mathrm{ng} / \mathrm{cm}^{2}$ on PEA and $\sim 600 \mathrm{ng} / \mathrm{cm}^{2}$ on PMA when $\mathrm{FN}$ is adsorbed from a $20 \mu \mathrm{g} / \mathrm{mL}$ solution).

To analyze the exposure and availability of integrin binding domains on $\mathrm{FN}$ following adsorption onto the polymeric surfaces, binding of conformation-specific monoclonal antibodies, in combination with an ELISA, was used. This is an established method to probe for structural or conformational changes of adsorbed proteins ${ }^{28,29}$ and can be used to measure FN activity. The selected antibodies recognized the central binding domain of FN, namely, HFN7.1. HFN7.1 is directed to the flexible linker between the 9th and 10th type III repeats of $\mathrm{FN}$ and has been identified as a receptor-mimetic probe for integrin binding and cell adhesion. ${ }^{13}$ In contrast, mAb1937 binds to the 8 th type III domain, ${ }^{14,30}$ therefore closer to the synergy domain (Fig. 1B). At very low concentration $(1 \mu \mathrm{g} / \mathrm{mL}), \mathrm{FN}$ biological activity is higher on PMA, as the binding is higher for both antibodies (Fig. 3B). However, increasing FN concentration to $20 \mu \mathrm{g} / \mathrm{mL}$ resulted in increased RGD accessibility on PEA that matched PMA levels, though binding of mAb1937 was significantly higher on PEA (Fig. 3B). Considering the change of FN activity with increasing FN concentration, there is a steady increase of the binding of both antibodies on PEA, while on PMA the binding of mAb1937 remains constant (Fig. 3B).

\section{Cell attachment and adhesion}

Short-term cell culture experiments were performed to investigate attachment and initial adhesion of L929 mouse fi- broblasts to the surfaces. Cell attachment to the different materials was measured using a standard protocol, allowing 20 min for attachment. ${ }^{22}$ The assay showed that the presence of an FN coating is essential for short-term cell attachment, as no cells were found on both bare polymer surfaces (Fig. 4A). When the polymers were coated with $\mathrm{FN}$ from a $20 \mu \mathrm{g} / \mathrm{mL}$ solution concentration $\left(\sim 600 \mathrm{ng} / \mathrm{cm}^{2}\right.$ according to Fig. 3A), cell attachment was similar on all surfaces ( $\sim 90 \%$ ), even if samples were washed extensively with DPBS before cell counting.

To study how cells responded to the FN-coated surfaces, $3 \mathrm{~h}$ adhesion experiments were performed in serum-free conditions after coating the surfaces from solutions containing $2,5,10$, and $20 \mu \mathrm{g} / \mathrm{mL}$ of FN. Cells were seeded at a low density $\left(5,000\right.$ cells $\left./ \mathrm{cm}^{2}\right)$ to maximize cell-material interactions and to minimize cell-cell interactions. Fibroblasts were found to spread on all surfaces, with the actin cytoskeleton becoming progressively more developed at increasing protein concentrations; at a minimum concentration of $2 \mu \mathrm{g} / \mathrm{mL}$, the actin cytoskeleton was not completely developed and only peripheral stress fibers were observed (Fig. 4C and Supplementary Fig. S2). The spreading area was similar on all surfaces for the different protein concentrations, with the exception of coatings from a $20 \mu \mathrm{g} /$ $\mathrm{mL}$ FN solution, where it was significantly higher on PEA (Fig. 4B).

Focal adhesion plaques were more developed on surfaces coated with higher FN concentration, where they were located at the end of prominent stress fibers (Fig. 5 and Supplementary Fig. S2). In order to quantify the maturation level of the focal adhesions on the different surfaces, frequency distributions for their size (defined as the length of the major axis of the focal adhesion plaque) were obtained through image analysis of the vinculin images (process detailed in Supplementary Fig. S3). Focal complexes (dot-like complexes shorter than $1 \mu \mathrm{m}^{12}$ ) were discarded from the analysis. The frequency distribution was similar on all surfaces, with a monotonic decrease from a higher fraction of smaller plaques. On PEA, the fraction of smaller focal plaques diminished with increasing FN concentration, as longer focal adhesions appeared. For example, the fraction of 
A

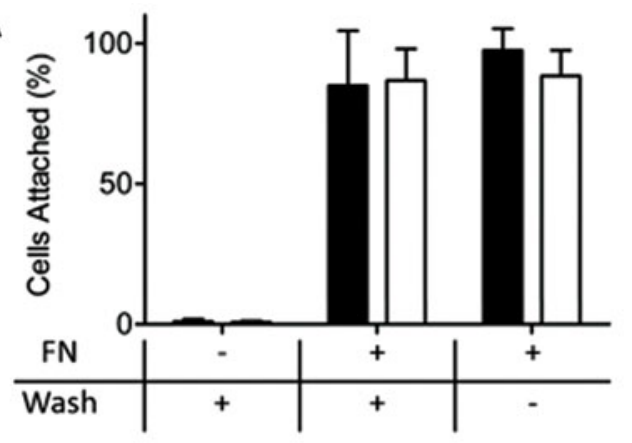

C
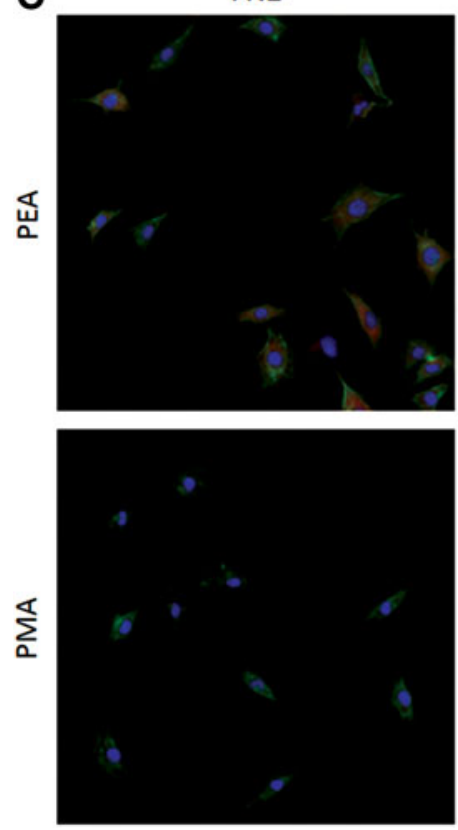

PEA PMA
B

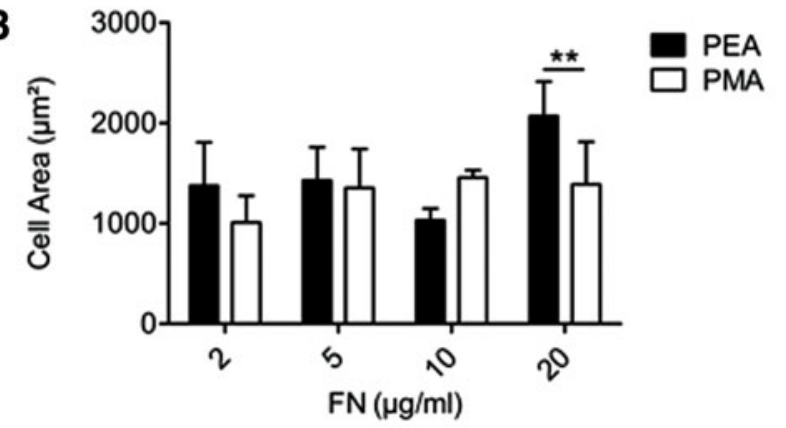

FN10
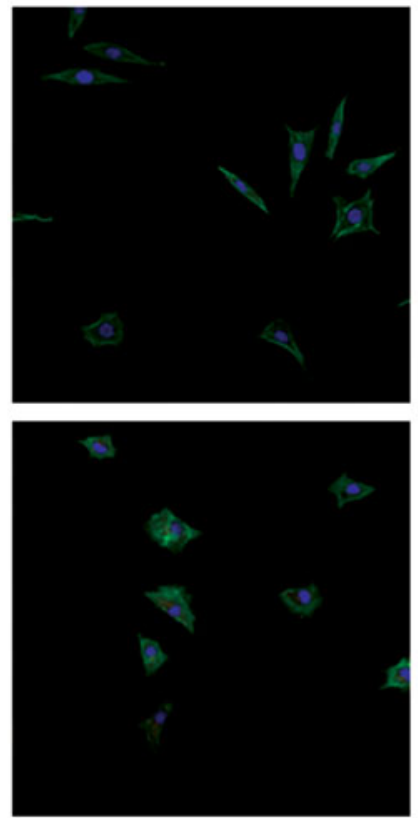

FN20
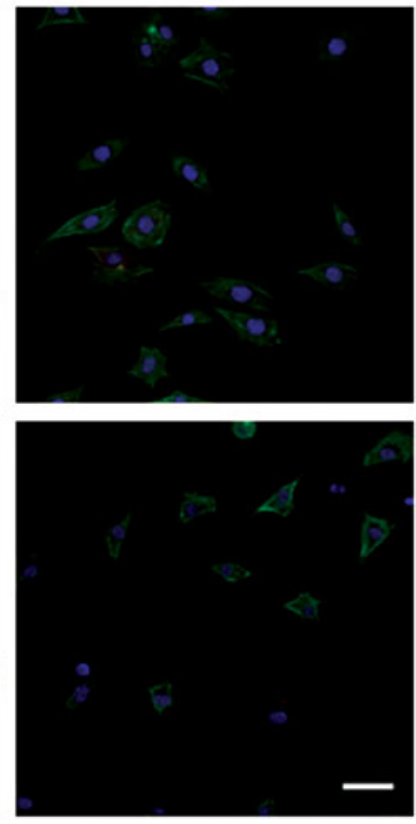

FIG. 4. Cell behavior on PEA and PMA coated with FN. (A) Attachment of L929 on PEA and PMA coated from a $20 \mu \mathrm{g} /$ mL FN solution. (B) Cell area on PEA and PMA coated from FN solutions of 2, 5, 10, and $20 \mu \mathrm{g} / \mathrm{mL}$. (C) Representative images of stained cells ("green" for actin, "red" for vinculin, and "blue" for nucleus) on PEA and PMA coated with 2, 5,10 , and $20 \mu \mathrm{g} / \mathrm{mL}$ of FN (denoted as FN2, FN5, FN10, and FN20, respectively). **p $<0.01$. Scale bar: $50 \mu \mathrm{m}$.

plaques longer than $2 \mu \mathrm{m}$ increased from $\sim 10 \%$ for an $\mathrm{FN}$ concentration of $2 \mu \mathrm{g} / \mathrm{mL}$ to $\sim 30 \%$ for a concentration of $20 \mu \mathrm{g} / \mathrm{mL}$ (Fig. 4). For PMA polymeric substrates the change in the frequency distribution was less pronounced (from $\sim 10 \%$ to $\sim 20 \%$ ).

This was confirmed by the median of the distribution; on PEA it showed a monotonic increase, while on PMA it remained constant (Fig. 6A). This trend of longer plaques became more apparent by using the focal adhesion area instead of length (Fig. 6A). By comparing the frequency distribution of the size of the focal adhesions to their respective area, it was evident that on PMA the plaques are prevalently dot-like compared to PEA, especially at lower FN concentration. As a refined measure of cell adhesion, the moment of inertia was used. This is a purely physical read out that examines the focal adhesion imprint relative to the center of mass of the distribution (Eq. 1). Overall, the moment of inertia of fibroblasts was significantly higher on $20 \mu \mathrm{g} / \mathrm{mL}$ FN-coated PEA surfaces compared to PMA (Fig. 6C).

\section{Cell adhesion after blocking RGD} and/or synergy domains of FN

The relevance of the RGD and the synergy domains on cell attachment and adhesion was studied by performing short-term cell culture assays on FN-coated samples after blocking these domains with HFN7.1 and mAb1937 monoclonal antibodies, respectively. For these studies, only surfaces coated with $20 \mu \mathrm{g} / \mathrm{mL}$ FN solutions were used. Blockage of the central binding domain of $\mathrm{FN}$ using HFN7.1 led to significantly reduced cell attachment of less than $10 \%$ (Fig. 7A). However, blocking FN with mAb1937 did not reduce the number of cells attached to either polymer. When both antibodies were employed, cell attachment on PMA was inhibited to the same levels (10\%) as for HFN7.1 blocking, while on PEA the percentage of attached cells was significantly higher (40\%). Next, we addressed the effect of blocking these $\mathrm{FN}$ domains on the number, size, and distribution of focal adhesions. Blocking with either HFN7.1 or mAb1937 (and both of them) significantly 
A
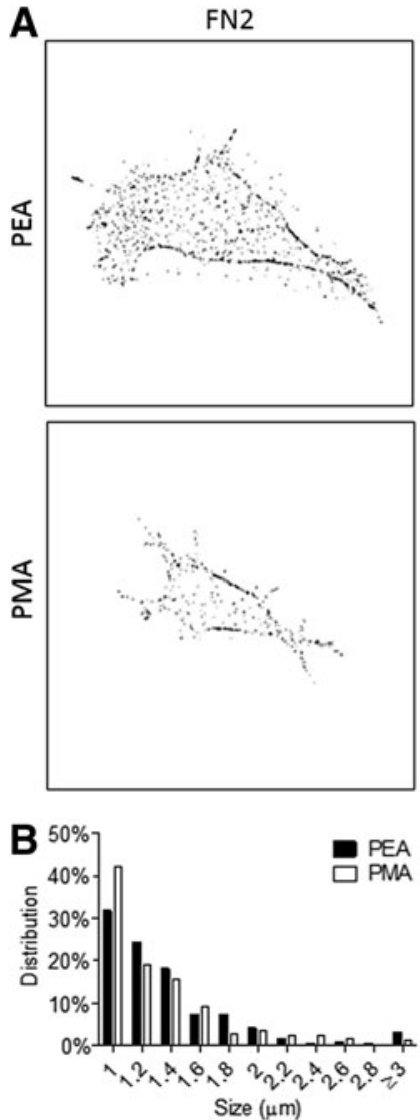
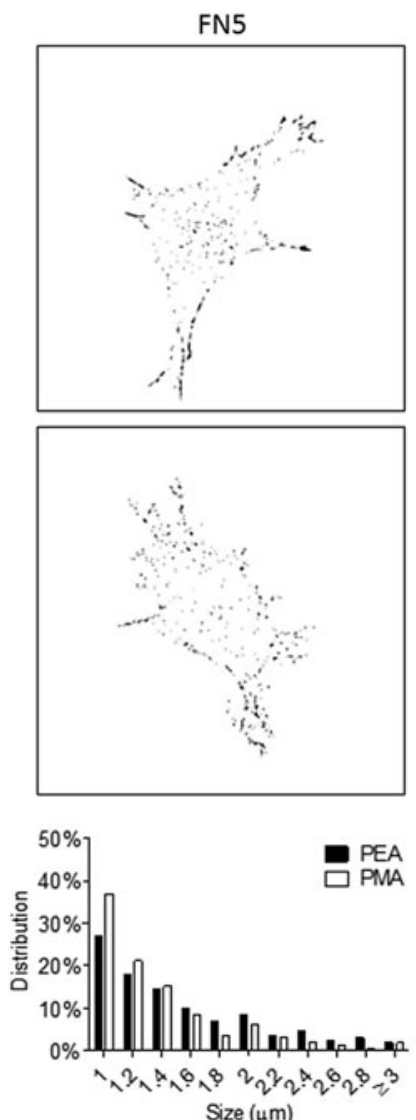

FN10
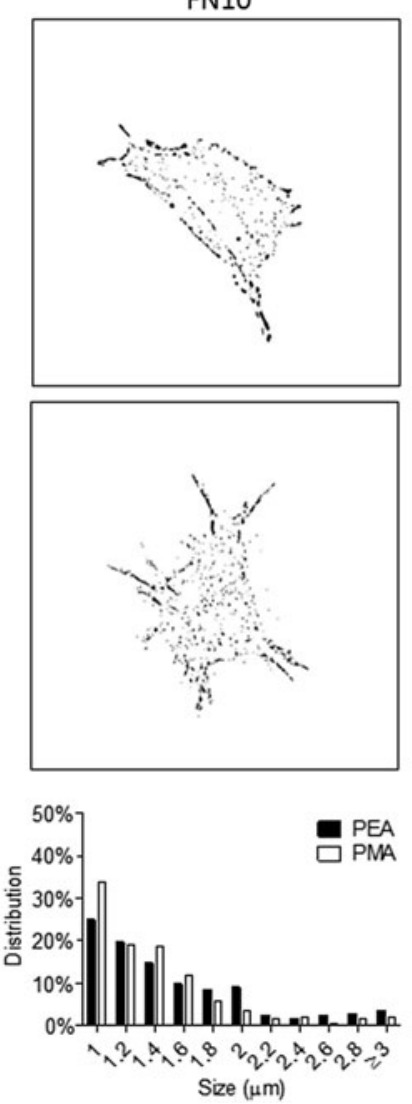
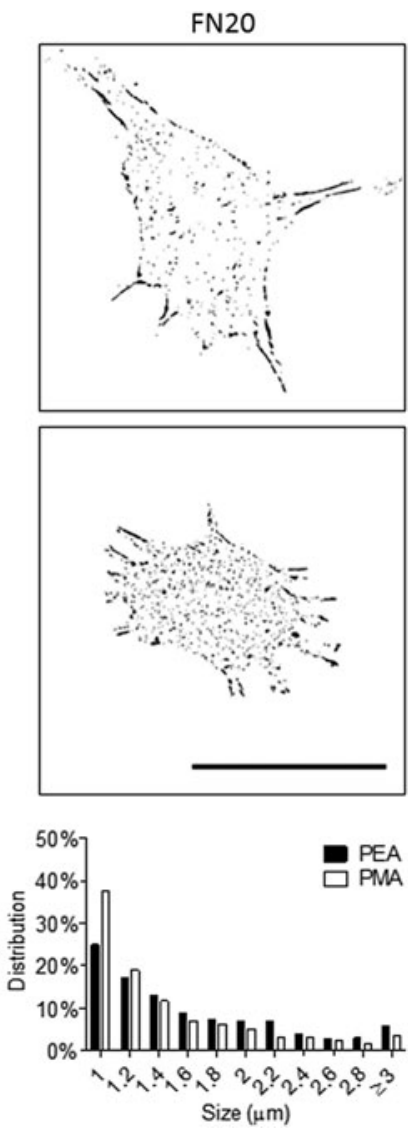

FIG. 5. Focal adhesion assembly on PEA and PMA coated with FN. (A) Representative inverted binary representation of focal adhesions of L929 on PEA and PMA coated with different concentrations of FN. (B) Images in (A) were quantified to build size distribution histograms of focal adhesions on both polymers. Scale bar: $50 \mu \mathrm{m}$.

diminished the number of focal adhesions on PMA, whereas a monotonic decrease was found on PEA (from HFN7.1 to mAb1937 and then both of them; Fig. 7B).

The same trend was found for the size of the corresponding focal adhesions, which were smaller on PEA after blocking with HFN7.1, and essentially absent after block- ing with mAb1937 or both antibodies (Fig. 7C). The size distribution of focal adhesions can be observed in Supplementary Figure S4. However, blocking with HFN7.1 did not alter the moment of inertia, and focal adhesions were still too far away from the center of the cell to end up in approximately the same value for this magnitude (Fig. 7D).
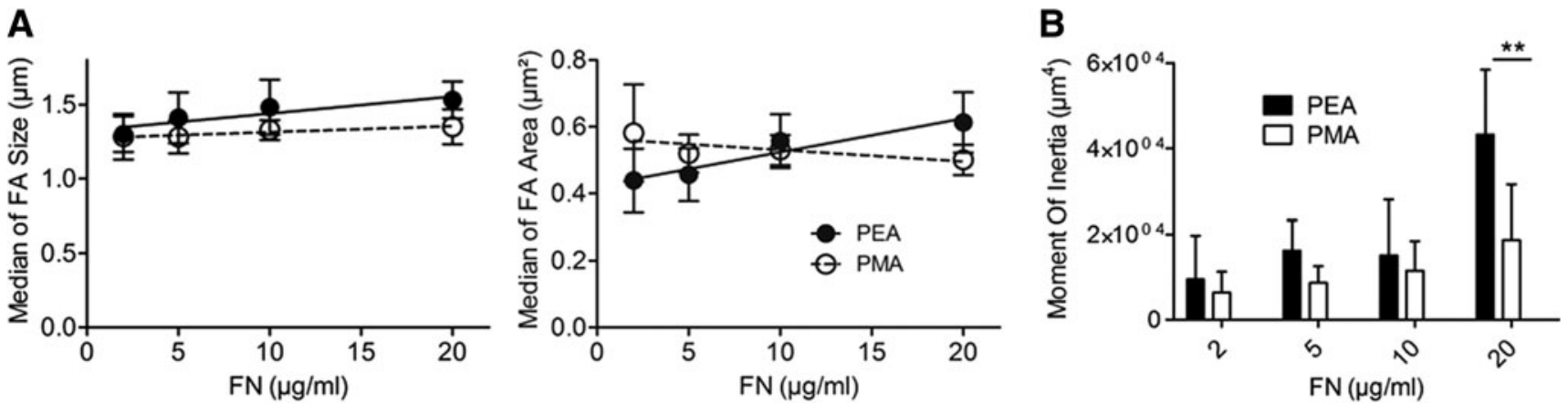

FIG. 6. Assessment of focal adhesions on PEA and PMA. (A) Median of focal adhesion size (left) and focal adhesion area on PEA and PMA coated with FN solutions of $2,5,10$, and $20 \mu \mathrm{g} / \mathrm{mL}$ (right). Note that the slope for PEA $(0.0101 x+0.00203)$ is statistically different from 0 for the median of focal adhesion area, which supports the increasing size of focal adhesions as the concentration of FN increases as well as the difference between focal adhesion size on PEA and PMA. $p<0.05$ ). (B) Moment of inertia for the distribution of focal adhesions calculated using Equation 1 for cells on PEA and PMA coated with different concentrations of FN. $* * p<0.01$. 

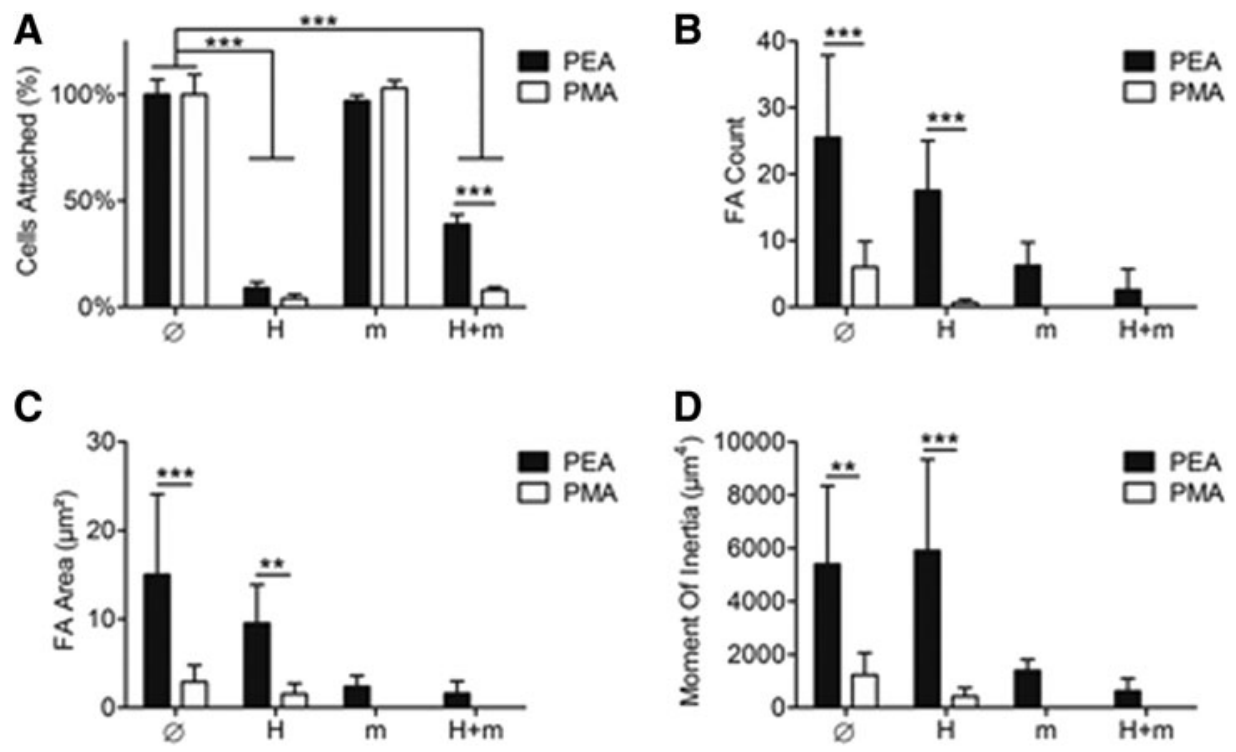

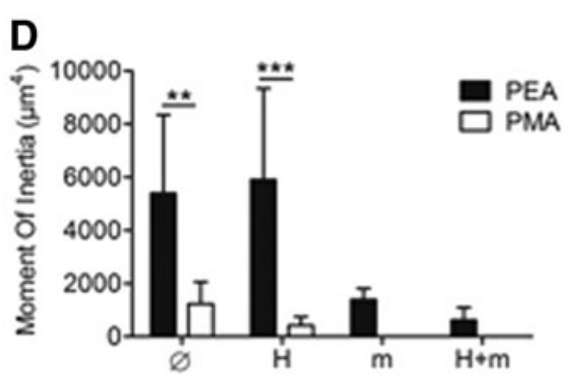

FIG. 7. Cell attachment and focal adhesion quantification after blocking RGD or synergy domain of FN. (A) Cells attachment on PEA and PMA after coating with an FN solution of $20 \mu \mathrm{g} / \mathrm{mL}$ and blocking FNIII $_{10}$ (HFN7.1, H), FNIII $_{9}$ $(\mathrm{mAb} 1937, \mathrm{~m})$, and both FNIII $_{9-}$ ${ }_{10}(\mathrm{H}+\mathrm{m}) ; \varnothing$ means no blocking. (B) Number of focal adhesions per cell, (C) total area occupied by focal adhesions on PEA and PMA, and (D) average moment of inertia per cell using the same blocking conditions. $* * p<0.01, * * * p<0.001$.
Conversely, using mAb1937 and the combination of both antibodies drastically decreased the number and size of focal adhesions and consequently the moment of inertia as well.

\section{Discussion}

It is well recognized that surface chemistry modulates FN adsorption in terms of the total amount of protein adsorbed and its conformation on the material surface. Seminal studies by Garcia et al. showed that by using model surface chemistries (SAMs with well-defined chemical groups, i.e., $-\mathrm{OH}$, $\left.\mathrm{CH}_{3}, \mathrm{NH}_{2}, \mathrm{COOH}\right)$ the integrin-binding domain of $\mathrm{FN}$ $\left(\mathrm{FNIII}_{7-10}\right)$ can be presented to cells with altered biological activity after adsorption on material surfaces. Modulation of FN activity at the material interface led to changes in integrin binding, organization of focal adhesions, and signaling that promoted cell differentiation with different efficiencies. $^{13,31,32}$ Focal adhesions are supramolecular structures that link adsorbed FN at the material interface with the actin cytoskeleton. Focal adhesion structure, size, composition, and dynamics depend on the information received from the surrounding matrix. Availability of key FN domains can promote integrin clustering, and this in turn can determine cell signaling and fate. ${ }^{33-35}$ Moreover, the composition, function, and size of focal adhesions have been correlated to each other: focal complexes $\sim 1 \mu \mathrm{m}$ are involved in migration and low-tension phenotypes that contain paxillin, vinculin, and phisphorylated proteins; focal adhesions $\sim 2-5 \mu \mathrm{m}$ are involved in intermediate tension phenotypes; supermature adhesions $>5 \mu \mathrm{m}$ are involved in high-tension phenotypes. $^{12,36}$

Here we show that FN conformation and distribution can be fine-tuned by using material surfaces with very similar chemical and physical chemistries. PEA and PMA consist of a vinyl chain with a side group that differs by only one methyl group (Fig. 2A). This subtle change in the underlying chemistry does not alter significantly the hydrophilicity of the surface (Fig. 2D) and both samples are sensed as simply rigid substrates by cells. ${ }^{24}$ In addition, the total amount of adsorbed FN on both PEA and PMA remained constant regardless the concentration of the adsorbing solution (Fig. 3A). However, the micro-/nanoscale distributions of FN differed significantly, with globular aggregates on PMA compared to an interconnected FN (nano) network on PEA (Fig. 2C). The different state of the adsorbed protein on the two polymers was also confirmed by dynamic contact angle measurements: contact angle hysteresis was significantly higher on FN-coated PEA due to a stronger decrease of the receding angles compared to PMA. This might suggest a higher protein surface coverage on PEA, compatible with the unfolding of the dimer arms and the formation of fibrils, compared to the maintenance of a globular conformation on PMA. Also, the extended conformation of FN on PEA might favor the molecular rearrangement of the protein in contact with water compared to the compact conformation on PMA.

The different FN presentation on the material surface has consequences at the molecular level for the availability of the integrin binding region of FN ( $\left.\mathrm{FNIII}_{9-10}\right)$. Importantly, after FN adsorption from a solution of concentration of $20 \mu \mathrm{g} / \mathrm{mL}$, the availability of the RGD domain remained constant for both PEA and PMA, whereas the synergy sequence (PHSRN) located at the $\mathrm{III}_{9}$ domain was preferentially available for cell engagement on PEA (Figs. 1C and 3 ). This has important consequences in terms of integrin binding and focal adhesion assembly. It has been shown that $\alpha_{5} \beta_{1}$ binding to $\mathrm{FN}$ requires both the RGD sequence $\left(\mathrm{FNIII}_{10}\right)$ and the synergy domain $\left(\mathrm{FNIII}_{9}\right)^{37,38}$ This observation also translated to cell adhesion on FN-coated PEA, where cell attachment occurred preferentially via $\alpha_{5} \beta_{1}$, in contrast to $\alpha_{\mathrm{v}} \beta_{3}$, which was mostly used for cells to adhere to $\mathrm{FN}$ adsorbed onto PMA. ${ }^{39}$

This biological response was triggered through FN presentation, which in turn was influenced by the underlying material surface. $^{21}$ We used vinculin as a marker of focal adhesions because it is recruited at adhesion sites where adhesion occurs via $\alpha_{5} \beta_{1}$ or $\alpha_{\mathrm{v}} \beta_{3}$ receptors. ${ }^{40}$ In addition, vinculin is required for myosin contractility-dependent adhesion strength and the coupling of cell area with traction 
force. $^{41}$ The formation (including size) of focal adhesions depends on the mechanical state of the local cell microenvironment. Stiff substrates and the application of mechanical inputs (stress and strain) involve the development of large focal adhesions, whereas soft substrates and the use of inhibitors of contractility favors the formation of focal-complexlike adhesions. ${ }^{42,43}$ Moreover, nanotopography-including nanopits, nanopillars, and nanogrooves-has been shown to alter the size and orientation of focal adhesions, including integrin clustering, focal adhesion size, and cytoskeleton organization. ${ }^{44,45}$

Here we show that conformation and distribution of FN on surfaces with very similar physicochemical properties (i.e., PEA and PMA) can be used to alter focal adhesion organization. Smaller focal adhesions were found on globular FN on PMA (focal complexes), whereas larger and better developed adhesions were quantified on PEA (Fig. 5). The size distribution of focal adhesions remained constant while the concentration of FN on the surface increased on PMA. However, on PEA, the distribution showed a higher fraction of larger focal adhesions as the concentration of FN on the surfaces increased (this can also be observed using the median-even if this is a poor representation of the whole distribution; Fig. 6). Larger focal adhesions on PEA and larger cells (Fig. 4B) resulted in higher values for the moment of inertia of the distribution of focal plaques on the surface (Fig. 6C). The stress applied by cells on substrates has been estimated to be $\sim 5.5 \mathrm{nN} / \mu \mathrm{m}^{2}{ }^{46}$ It is then suggested that cells are better positioned to exert larger forces on FN fibrils assembled on PEA than on globular FN adsorbed on PMA, even if both surfaces are sensed as rigid by cells ( $\mathrm{E}$ above $1 \mathrm{MPa}$ for both of them).

Blocking experiments with monoclonal antibodies directed against FNIII $_{10}$ (HFN7.1) and FNIII $_{9}$ (mAb1937) further supported the notion that PEA and PMA induced a polymer-specific biological response. Blocking FNIII $_{10}$ (which prevents the availability of RGD) drastically diminished the number of cells on both surfaces, an effect not observed when blocking $\mathrm{FNIII}_{9}$ (synergy sequence) (Fig. 7A). Therefore, when the synergy sequence of FN (PHSRN) was not available for interaction (on PEA), cells were still able to adhere using only the RGD domain (and $\alpha_{\mathrm{v}} \beta_{3}$ rather than $\left.\alpha_{5} \beta_{1}\right){ }^{37}$ Likewise, the number and size of focal adhesions was significantly diminished on PMA after blocking with either antibody, whereas a significant number of focal adhesions (but smaller) were still found on PEA after blocking each one of the domains, or both simultaneously (Fig. 7 and Supplementary Fig. S4). This result directly correlated with the extended conformation of FN molecules on PEA (that leads to the organization into nanonetworks observed by AFM; Fig. 2) and the globularlike shape of the protein on PMA. Even after blocking FNIII $_{9-10}$, other domains were still available for integrin binding on PEA (e.g., $\mathrm{FNI}_{5}$ and the variable region) but not on PMA, as a change in the conformation of the molecule (fibrillogenesis) was required for these domains to be exposed (Fig. 1D). ${ }^{47}$ Overall, we present a mechanistic model for the different conformation of $\mathrm{FN}$ induced by the underlying material surface that accounts for the availability of the integrin binding domains ( FNIII $\left._{9-10}\right)$ and the effect of blocking antibodies (HFN7.1, mAb1937) as discussed (Fig. 1).

\section{Conclusions}

We have shown that FN conformation can be triggered by material surfaces with very similar chemical composition (PEA and PMA differ in only one methyl group in the side chain) and physical properties (wettability and charge). FN is organized in nanonetworks on PEA, while a globular conformation was observed on PMA. As a consequence, FN molecules were adsorbed in an extended conformation on PEA, which provides better availability of the synergy sequence and other integrin-binding domains of the molecule (e.g., $\mathrm{FNI}_{5}$ ). The distinct organization of FN on PEA led to a higher fraction of larger focal adhesions, whereas smaller focal-complex points were observed on PMA. The maintenance of focal adhesions, even after blocking $\mathrm{FNIII}_{9-10}$ with monoclonal antibodies on PEA, but not on PMA, confirmed the different conformation of $\mathrm{FN}$ on these polymeric surfaces. Our results suggest that cells are highly sensitive to the organization of FN on material surfaces regardless of the properties of the underlying polymeric coating. In summary, PEA and PMA can be used as a simple material platform to fine-tune the microenvironment presented to cells by controlling the intermediate layer of protein adsorbed onto the substrates. This material-based modulation of $\mathrm{FN}$ is a versatile tool to study and understand cell behavior in biomedical research, including tissue engineering and disease biology.

\section{Acknowledgment}

The support from ERC through HealInSynergy 306990 is acknowledged.

\section{Author Disclosure Statement}

The authors declare no competing financial interests exist.

\section{References}

1. Sipe JD. Tissue engineering and reparative medicine. In: Reparative Medicine: Growing Tissues and Organs. Sipe JD, Kelley CA, McNichol LA, (eds.) New York Academy of Sciences: New York, NY; pp. 1-9; 2002.

2. Griffith LG, Naughton G. Tissue engineering - current challenges and expanding opportunities. Science. 2002;295: 1009-1014.

3. Pompe T, Markowski M, Werner C. Modulated fibronectin anchorage at polymer substrates controls angiogenesis. Tissue Eng. 2004;10:841-848.

4. Werner C, Garcia AJ. Interfaces to control cell-biomaterial adhesive interactions. In: Polymers for Regenerative Medicine. Werner C, (ed.) Springer: Berlin; pp. 171-190; 2006.

5. Salmerón-Sánchez M, Altankov G. Cell-protein-material interaction in tissue engineering. In: Tissue Engineering. Eberli D, (ed.) InTech: Vienna, Austria; pp. 77-102; 2010.

6. Gumbiner BM. Cell adhesion: the molecular basis of tissue architecture and morphogenesis. Cell. 1996;84:345-357.

7. Erickson HP, Carrell NA, McDonagh J. Fibronectin molecule visualized in electron microscopy: a long, thin, flexible strand. J Cell Biol. 1981;91:673-678.

8. Erickson HP, Carrell NA. Fibronectin in extended and compact conformations. Electron microscopy and sedimentation analysis. J Biol Chem. 1983;258:14539-14544.

9. Pankov R, Yamada KM. Fibronectin at a glance. J Cell Sci. 2002;115:3861-3863. 
10. Leahy DJ, Aukhil I, Erickson HP. 2.0 A crystal structure of a four-domain segment of human fibronectin encompassing the RGD loop and synergy region. Cell. 1996;84: $155-164$.

11. Hynes RO. Integrins: bidirectional, allosteric signaling machines. Cell. 2002;110:673-687.

12. Geiger B, Bershadsky A, Pankov R, et al. Transmembrane crosstalk between the extracellular matrix-cytoskeleton crosstalk. Nat Rev Mol Cell Biol. 2001;2:793-805.

13. Keselowsky BG, Collard DM, García AJ. Surface chemistry modulates fibronectin conformation and directs integrin binding and specificity to control cell adhesion. J Biomed Mater Res A. 2003;66:247-259.

14. Michael KE, Vernekar VN, Keselowsky BG, et al. Adsorptioninduced conformational changes in fibronectin due to interactions with well-defined surface chemistries. Langmuir. 2003; 19:8033-8040.

15. Toworfe GK, Composto RJ, Adams CS, et al. Fibronectin adsorption on surface-activated poly(dimethylsiloxane) and its effect on cellular function. J Biomed Mater Res A. 2004;71:449-461.

16. Baugh L, Vogel V. Structural changes of fibronectin adsorbed to model surfaces probed by fluorescence resonance energy transfer. J Biomed Mater Res A. 2004;69:525-534.

17. Grinnell F, Feld MK. Fibronectin adsorption on hydrophilic and hydrophobic surfaces detected by antibody binding and analyzed during cell adhesion in serum-containing medium. J Biol Chem. 1982;257:4888-4893.

18. Altankov G, Thom V, Groth T, et al. Modulating the biocompatibility of polymer surfaces with poly(ethylene glycol): effect of fibronectin. J Biomed Mater Res. 2000;52: 219-230.

19. Teichmann J, Morgenstern A, Seebach J, Schnittler HJ, Werner C, Pompe T. The control of endothelial cell adhesion and migration by shear stress and matrix-substrate anchorage. Biomaterials. 2012;33:1959-1969.

20. Cantini M, González-García C, Llopis-Hernández V, et al. Material-driven fibronectin fibrillogenesis. In: Proteins at Interfaces III State of the Art. Horbett T, Brash JL, Norde W, (eds.) American Chemical Society: Washington, DC; pp. 471-496; 2012.

21. Salmerón-Sánchez M, Rico P, Moratal D, et al. Role of material-driven fibronectin fibrillogenesis in cell differentiation. Biomaterials. 2011;32:2099-2105.

22. Humphries MJ. Cell adhesion assays. In: Methods in Molecular Biology, Extracellular Matrix Protocols. Even-Ram S, Artym VA, (eds.) Humana Press: New York, NY; pp. 203210; 2009.

23. Berginski ME, Gomez SM. The Focal Adhesion Analysis Server: a web tool for analyzing focal adhesion dynamics. F1000Res. 2013;2:68.

24. González-García C, Moratal D, Oreffo RO, et al. Surface mobility regulates skeletal stem cell differentiation. Integr Biol (Camb). 2012;4:531-539.

25. Van Damme HS, Hogt AH, Feijen J. Surface mobility and structural tensions of poly(n-alkyl methacrylates) probed by dynamic contact angle measurements. J Colloid Interface Sci. 1989;114:167-172.

26. Takahashi S, Kesemura T, Asano K. Surface molecular mobility for copolymers having perfluorooctyl and/or polyether side chains via dynamic contact angle. Polymer. 1997;38: 2107-2111.

27. Gugutkov D, González-García C, Rodríguez Hernández JC, et al. Biological activity of the substrate-induced fibronectin network: insight into the third dimension through electrospun fibers. Langmuir. 2009;25:10893-10900.

28. Ugarova TP, Zamarron C, Veklich Y, et al. Conformational transitions in the cell binding domain of fibronectin. Biochemistry. 1995;34:4457-4466.

29. McClary KB, Ugarova T, Grainger DW. Modulating fibroblast adhesion, spreading, and proliferation using selfassembled monolayer films of alkylthiolates on gold. J Biomed Mater Res. 2000;50:428-439.

30. Schoen RC, Bentley KL, Klebe RJ. Monoclonal antibody against human fibronectin which inhibits cell attachment. Hybridoma. 1982;1:99-108.

31. Keselowsky BG, Collard DM, García AJ. Surface chemistry modulates focal adhesion composition and signaling through changes in integrin binding. Biomaterials. 2004;25: 5947-5954.

32. Keselowsky BG, Collard DM, García AJ. Integrin binding specificity regulates biomaterial surface chemistry effects on cell differentiation. Proc Natl Acad Sci USA. 2005;102: 5953-5957.

33. Wehrle-Haller B. Structure and function of focal adhesions. Curr Opin Cell Biol. 2012;24:116-124.

34. Hanein D, Horwitz AR. The structure of cell-matrix adhesions: the new frontier. Curr Opin Cell Biol. 2012;24: 134-140.

35. Schiller HB, Fässler R. Mechonasensitivity and compositional dynamics of cell-matrix adhesions. EMBO Rep. 2013;14:509-519.

36. Biggs MJ, Richards RG, Gadegaard N, et al. The use of nanoscale topography to modulate the dynamics of adhesion formation in primary osteoblasts and ERK/MAPK signalling in STRO-1 + enriched skeletal stem cells. Biomaterials. 2009;30:5094-5103.

37. Mould AP, Askari JA, Aota S, et al. Defining the topology of integrin alpha5beta1-fibronectin interactions using inhibitory anti-alpha5 and anti-beta1 monoclonal antibodies. Evidence that the synergy sequence of fibronectin is recognized by the amino-terminal repeats of the alpha5 subunit. J Biol Chem. 1997;272:17283-17292.

38. García AJ, Schwarzbauer JE, Boettiger D. Distinct activation of alpha5beta1 integrin show differential binding to RGD and synergy domains of fibronectin. Biochemistry. 2002;41:9063-9069.

39. Llopis-Hernández V, Rico P, Moratal D, et al. Role of material-driven fibronectin fibrillogenesis in protein remodeling. Biores Open Access. 2013;2:364-373.

40. Galbraith CG, Yamada KM, Sheetz MP. The relationship between force and focal complex development. J Cell Biol. 2002;159:695-705.

41. Dumbauld DW, Lee TT, Singh A, et al. How vinculin regulates force transmission. Proc Natl Acad Sci USA. 2013;110:9788-9793.

42. Geiger B, Spatz JP, Bershadsky AD. Environmental sensing through focal adhesions. Nat Rev Mol Cell Biol. 2009; 10:21-33.

43. Chan CE, Odde DJ. Traction dynamics of filopodia on compliant substrates. Science. 2008;322:1687-1691.

44. Yim EK, Darling EM, Kulangara K, et al. Nanotopographyinduced changes in focal adhesions, cytoskeletal organization, and mechanical properties of human mesenchymal stem cells. Biomaterials. 2010;31:1299-1306.

45. Dalby MJ, Gedegaard N, Oreffo RO. Harnessing nanotopography and integrin-matrix interactions to influence stem cell fate. Nat Mater. 2014;13:558-569. 
46. Balaban NQ, Schwarz US, Riveline D, et al. Force and focal adhesion assembly: a close relationship studied using elastic micropatterned substrates. Nat Cell Biol. 2001;3:466-472.

47. Leiss M, Beckmann K, Girós A. The role of integrin binding sites in fibronectin matrix assembly in vivo. Curr Opin Cell Biol. 2008;20:502-507.

Address correspondence to: Prof. Manuel Salmerón-Sánchez Division of Biomedical Engineering School of Engineering University of Glasgow

Rankine Building, Oakfield Avenue Glasgow G12 8LT United Kingdom

E-mail: manuel.salmeron-sanchez@glasgow.ac.uk

Marco Cantini, PhD Division of Biomedical Engineering

School of Engineering

University of Glasgow

Rankine Building, Oakfield Avenue

Glasgow G12 8LT

United Kingdom

E-mail: marco.cantini@glasgow.ac.uk

\section{Abbreviations Used}

$\mathrm{ACA}=$ advancing contact angle

$\mathrm{AFM}=$ atomic force microscopy

DPBS $=$ Dulbecco's phosphate buffered saline

$\mathrm{ECM}=$ extracellular matrix

ELISA = enzyme-linked immunosorbent assay

$\mathrm{FBS}=$ fetal bovine serum

$\mathrm{FN}=$ fibronectin

$\mathrm{PEA}=$ poly(ethyl acrylate $)$

PMA $=$ poly(methyl acrylate)

$\mathrm{RCA}=$ receding contact angle

SAMs $=$ self-assembled monolayers

$\mathrm{SCA}=$ static contact angle 